\title{
Regards croisés sur une fin annoncée
}

François-André Isambert, Denis Pelletier et Grace Davie

\section{(2) OpenEdition}

Journals

Édition électronique

URL : http://journals.openedition.org/assr/2160

DOI : $10.4000 /$ assr. 2160

ISSN : $1777-5825$

Éditeur

Éditions de l'EHESS

Édition imprimée

Date de publication : 1 avril 2004

Pagination : 5-16

ISBN : 2-222-96746-5

ISSN : 0335-5985

Référence électronique

François-André Isambert, Denis Pelletier et Grace Davie, « Regards croisés sur une fin annoncée », Archives de sciences sociales des religions [En ligne], 126 | avril - juin 2004, mis en ligne le 17 novembre 2005, consulté le 30 avril 2019. URL : http://journals.openedition.org/assr/2160 ; DOI : 10.4000/ assr.2160 
Arch. de Sc. soc. des Rel., 2004, 126, (avril-juin 2004) 5-16

François ISAMBERT

Denis PELLETIER

Grace DAVIE

\title{
REGARDS CROISÉS SUR UNE FIN ANNONCÉE
}

\author{
À propos de : \\ HERVIEU-LÉGER (Danièle), Catholicisme, la fin d'un \\ monde. Paris, Bayard, 2003, 336 p.
}

\section{Les trois âges du catholicisme français}

Il y a longtemps que les sociologues du catholicisme parlent d'un recul de celui-ci, du moins en France. Mais il est plus rare de voir situer cette régression dans sa phase terminale «Fin d'un monde », c'est diagnostiquer une mort, et parler d' " un monde », ce n'est pas seulement emprunter une expression popularisée par Émile Poulat, c'est insister sur le caractère global (on pourrait dire «total » avec Marcel Mauss) de cette fin. Pour une expertise aussi radicale, on doit en préciser la portée. Et si un petit reproche doit être fait à D.H.-L. c'est de partir de but en blanc: «Les catholiques français sont-ils mal traités dans la société française ?». En fait, c'est tantôt les catholiques, tantôt l'Église comme Institution, tantôt « le christianisme » dont on rappelle qu'ils ont perdu tout ou partie de leur crédit. Qu'est-ce à dire ? L'auteure ne cède pas à la tentation d'utiliser ce point de départ pour faire le procès de la « ringardise » d'une Église qui, au moins en France, est « digne d'estime» (et il n'est question ici que de la France, à la fois «France laïque » et «Fille aînée de l'Église ») et a fait des efforts sérieux pour adapter sa pastorale au monde d'aujourd'hui. Mais le mal est plus profond. Le catholicisme d'aujourd'hui, malgré l'ouverture d'esprit d'une grande part de son clergé, paraît mal accordé avec la modernité de ce monde. Alors, de quoi s'agit-il ? D'un archaïsme inhérent à la structure et à la prédication catholiques, à une crise de la société en tant que telle, ou à une simple différence de tempo entre deux évolutions ? Ces différentes hypothèses - pour autant qu'elles s'excluent - sont en fait abordées, avec une conclusion pratique qui fait appel autant aux convictions de l'A. qu'aux faits analysés. Tout l'ouvrage tournant autour de la notion de "modernité », elle se devait d'en préciser au préalable les étapes. 
La dernière est évidemment celle d'un nouveau paganisme. Le mot «païen », exhumé par France, pays de mission? (1943) et repris par Mgr Simon cinquante ans plus tard (Vers une France païenne? [Cana, 1999] [cf. Arch. 112.102]) était destiné à faire tomber l'illusion d'une France à substrat culturel chrétien, qu'ont cru retrouver les tenants d'un catholicisme populaire. Mais que pouvait-on mettre sous le terme de «païen»? Pas cette religiosité foisonnante dont Marc Augé s'est fait le chantre et qui rejoint par bien des points le «polythéisme » de Max Weber. Pour les deux premiers auteurs (Godin et Daniel) «la déchristianisation attestée dans un certain nombre de régions est indissociablement liée à un délabrement sociétal et moral ». (Jacques Valdour, dans sa série La Vie ouvrière, 17 vol. 19141934, avait brossé un tableau encore plus inquiétant, mais dont le retentissement avait été bien moindre). "Paganisme» est alors synonyme de «vide social». Il n'en est pas de même pour Mgr Simon qui, comme beaucoup de catholiques, a lu Le Désenchantement du monde de Marcel Gauchet, pour qui toutes les valeurs rassemblées sous le terme de «laïcité », même si elles s'essoufflent un peu, forment une base éthico-politique sur laquelle fonctionne la société civile d'aujourd'hui en mettant entre parenthèse toute religion. La difficulté est que cette laïcité implique un pluralisme, une « reconnaissance » (je dirais une «tolérance ») qui se désarment eux-mêmes comme principes créatifs. Dans la perspective de D.H.-L., cette sorte de paganisme est le point d'aboutissement d'un processus, si bien qu'on peut tracer plusieurs «âges d'une mutation ».

a) La «laïcisation », passage du monopole ecclésial de toute autorité, doctrinale, politique, morale et scientifique, à la constitution d'un secteur séculier, gouverné par le bon sens et la « raison ». C'est l'œuvre des «Lumières » à la française, de la constitution, par étapes de la République sans référence religieuse, et de la souveraineté de la vérité scientifique. Mutation non sans opposition religieuse et politique. La radicalisation du conflit partageait certes «les deux France ». Mais aussi elle divisait profondément le camp des «laïcs» d'une part, libéraux contre anti-cléricaux et celui des catholiques d'autre part, les uns acceptant en gros la laïcité et se faisant taxer de «modernistes » par les «intransigeants ». La laïcisation qui se drape dans l'histoire de la Révolution proclame comme valeur-clé la liberté de penser, donc celle de croire ou de ne pas croire.

b) La «sécularisation interne», caractérisée par «le travail interne de la modernité dans la sphère religieuse ». C'est le message de l'Église qui doit être traduit en termes communs - certains diront «profanes ». Toute l'action des catholiques est repensée en termes de propagande ou de stratégie. C'est ce que craignaient le plus les antimodernistes. Ce fut l'enjeu de luttes incessantes à l'intérieur du catholicisme, de sa théologie, des Facultés catholiques, du clergé lui-même avec la crise des «prêtres-ouvriers ». L'accomplissement de cette transformation sembla s'effectuer avec le concile Vatican II qui, pourtant, fut remis en question sur bien des points avec ce que Paul Ladrière appelle Le Retour des certitudes (1987).

c) Le temps de l' «ultramodernité » pose au lecteur un problème sémantique. Certains avaient déjà acclimaté le terme «postmoderne », non sans une ambiguïté que l'on pourrait appeler «dialectique ». Ce que l'on s'est généralement accordé pour nommer « modernité », c'est-à-dire le triomphe de l'esprit scientifique et technique et la condamnation des préjugés, le développement d'une sphère privée des croyances et des mœurs et une autonomie du jugement a été considérée par certains comme «dépassée ». Mais pour les uns, il s'agit de pousser plus avant en 
se débarrassant des lambeaux d'hypocrisie que traîne avec elle la morale bourgeoise, ce qui conduit à un libéralisme généralisé. Pour les autres, la postmodernité est le contre-pied du raisonnable dans tous les domaines, l'exaltation de l'antiscience ou des para-sciences, ou encore la réhabilitation du sadisme, ou une esthétique de l'horrible, voire le Requiem pour les Twin Towers (dans Power inferno de Jean Baudrillard, Paris, Galilée, 2002 [coll. «L'espace critique»]). L'ultramodernité s'approcherait plus de la première que de la seconde de ces deux versions de la postmodernité (encore que certaines ressources de l'irrationalisme puissent se mettre à son service). C'est la "remise en question des fondements de toute «absoluité » (p. 88). »Ces trois phases sont à la fois qualitativement distinctes et étapes d'un même processus, aboutissant à un individualisme incompatible avec l'esprit et la structure du catholicisme. Comme on le voit, c'est la société qui a changé en profondeur, au point que les points forts qui servaient d'appui au catholicisme, à savoir la ruralité et la famille, l'ont « lâché ».

À chacune de ces phases, le détachement à l'égard du catholicisme augmente. Ce sont aussi des phases d'urbanisation. La consubstantialité séculaire de l'Église et de la ruralité a été vue avec une pertinence particulière par Le Bras (L'Église et le village, 1976). Si on a pu proposer un instrument de mesure, le degré de la pratique cultuelle dans une population censée catholique, l'inventeur même de cette métrique, Gabriel Le Bras, était le premier à en dénoncer le caractère relatif. Ses recherches sur les visites pastorales des siècles passés l'amenaient à penser qu'il fallait sans doute opposer moins la France d'autrefois à celle de maintenant, que des zones d'attachement ou de détachement divers à l'Église, d'où l'importance de la cartographie que développa le chanoine Boulard et ses successeurs. Or, les aires urbaines paraissant dans l'ensemble plus décatholicisées, les progrès de l'urbanisation engendreraient par là même une baisse d'emprise de l'Église. On trouve là un évolutionnisme de fait, à base démographique et que personne ne nie. Mais à lui tout seul, il laisserait croire que les campagnes seraient naturellement religieuses et les villes naturellement profanes. Il était donc à la fois courageux et essentiel de s'attaquer à une image idéalisée de la France rurale-et-catholique, ce qui donne la partie la plus forte de l'ouvrage, analysant une transformation des esprits et des comportements chez les ruraux, avec référence à Yves Lambert et à son Dieu change en Bretagne La religion à Limerzel de 1900 à nos jours (Paris, Cerf, 1985 [coll. "Sciences Humaines »]). Ici, il n'est guère question de polémiques. Certes, la modernisation de la liturgie et de la pastorale rencontre bien des résistances et les générations sont en fréquent désaccord sur les croyances et les rites traditionnels mais «le délaissement progressif des temps clos» (op.cit., p. 322) s'est effectué en définitive sans drame et Y. Lambert ne croit pas que ce soit la source principale de la désaffection des populations rurales.

En revanche, « on peut dire que les années 1960 sont celles où le catholicisme français secoue définitivement ses adhérences à un univers rural lui-même révolu » (p. 113). Depuis les années trente, déjà, la JAC avait compris l'inéluctabilité de la professionnalisation du monde agricole, cela non sans conflits dans l'Église, mais avec l'appui d'une partie dynamique du clergé, luttant contre le dépérissement social des campagnes. Que ce réformisme ait été vécu comme tension entre des valeurs traditionnelles - la terre - et un appétit de modernisation, on le comprend bien, mais l'A. va jusqu'à voir là une attitude contradictoire, minée de l'intérieur par l'incompatibilité entre un « intransigeantisme » et un « modernisme ». C'est ce qui fait à la fois l'originalité et une certaine fragilité de son interprétation. La JAC, 
comme toute l'Action Catholique des jeunes a contribué à développer un christianisme militant, résolument christocentrique, tourné vers le monde pour le transformer et l'animer par un réseau de relations sociales dominées par l'esprit de solidarité. Les coopératives ont pour mission de traduire en actes le Sermon sur la Montagne. Certes, Ulrich Beck peut bien parler d'une « religion séculière de l'Amour », tendant à prendre la place du christianisme, mais c'est le christianisme lui-même qui, en devenant apologie du Christ, a récolté partiellement les effets des tendances personnalistes et affectivistes de l'avant-guerre. On peut en dire autant du militantisme : en un sens, l'apôtre qui devient militant se sécularise, mais il peut prétendre - et on ne peut d'emblée lui donner tort - qu'il a intégré dans son christianisme un dynamisme dont seul, jusque-là, le militant communiste faisait preuve. L'analyse de D.H.-L. puise incontestablement aux sources du wébérisme. On pourrait donner pour titre à son analyse : La conviction catholique et l'esprit de la ruralité. Mais, contrairement à Weber, ce dont il s'agit n'est pas de fonder l'une dans l'autre; bien plutôt de fonder les transformations de l'une dans celles de l'autre. Le rattachement avoué du lien entre cette ruralité archétypique et le catholicisme, à une «affinité élective » nous fait entrer dans une problématique où la régulation de la vie se fait en consonance sur le mode économique et sur le mode religieux. L'agriculteur, devenu ingénieur de la terre, ne peut plus penser le monde comme son ancêtre qui attendait les bienfaits - ou les méfaits - du ciel. La rationalisation de sa comptabilité ne fait pas bon ménage avec la vision de sa propre prospérité comme une bénédiction divine.

Selon un schéma analogue, la famille, autre point d'appui du catholicisme, se modifie radicalement à partir du moment où elle devient le lieu des relations affectives réussies et non plus la cellule sacrale de l'indissolubilité des liens conjugaux et parentaux. Même l'Église préfère adapter une pastorale pour divorcés plutôt que de fulminer des excommunications. Cette dépréciation relative de l'Autorité au profit de la spontanéité n'est pas seulement un ébranlement de tout l'édifice institutionnel, c'est une remise en question des fondements de la Foi. Dans le principe séculaire du magistère, le contenu de la Foi est tout entier objet d'enseignement et elle-même est d'abord obéissance. D'où le fait que des principes moraux, des doctrines théologiques et même des théories scientifiques puissent être condamnés et que ces condamnations aient un sens. Explorant les convictions des « sans religion » dans un pays de culture chrétienne, Sylvette Denèfle (Sociologie de la sécularisation. Etre sans-religion en France à la fin $d u \quad X X^{e}$ siècle [Paris, L'Harmattan, 1997] [cf. Arch. 122.63]) rejoint le point de vue ici développé d'une aspiration assez générale à l'autonomie du jugement, tant moral que politique et scientifique. Or, sur ce dernier plan, où l'Église semble ne pouvoir mener que des combats d'arrière-garde, un réveil du dogmatisme s'est effectué dans le domaine biologique. Un nouvel espace sacré a été tracé autour des phénomènes vitaux, interdisant par là même une libre recherche à leur sujet, renforçant la clôture autour des comportements sexuels. Selon l'A. l'Église est prisonnière ici d'une certaine idée de la nature comme norme. Alors que de nombreux fidèles et prêtres voudraient dédramatiser les interdits au sujet de l'homosexualité, une sorte de mécanisme interne entraîne les représentants de l'Église sur des positions contre le PACS. Là, on peut vraiment dire que la troupe ne suit plus.

Finalement, ce qui est en question c'est un nouvel individualisme. Plaçant le JE au centre du dispositif des relations entre les individus et entre ceux-ci et le monde, la civilisation actuelle lance une nouvelle course au bonheur. Celle-ci est polarisée 
entre d'une part l'aspiration vers des formes d'équilibre psychique soumises à des traitements de plus en plus sophistiqués, d'autre part le risque de manquer, d'évidence planétaire. Cela entraîne une transformation éthique majeure : c'est l' «accomplissement de soi » qui devient la clé de la Wertrationalität nouvelle. En un sens, s'accomplir, c'est trouver le démon personnel auquel Max Weber recommandait d'obéir. C'est trouver un calling intérieur plus intime et plus impératif à la fois que les commandements de l'autorité religieuse. C'est aussi trouver la direction dans laquelle on va œuvrer pour combler les manques qu'on aura décelés. D'où la multiplication des bénévolats en tous genres. D'où aussi cette préoccupation de la sauvegarde de la planète et la vigilance à l'égard de la détérioration du milieu. Sans doute voit-on ici affleurer cette idée que le catholicisme a réussi à injecter dans la culture de base, à savoir la méfiance à l'égard de tout dérangement de l'ordre naturel. Mais cette idée est vite emportée par un progrès scientifique qui, justement, franchit les anciennes frontières et met en cause les concepts qui les justifiaient. Or cette éthique du sujet est incompatible, nous dit l'A. avec le catholicisme. Dans un chapitre essentiel, D.H.-L. prend à bras-le-corps cette «ultramodernité » qui voit dans notre satisfaction et celle du plus grand nombre un objectif, sans doute immensément distant, mais accessible sans rupture avec notre univers.

Ce que D.H.-L. ne dit pas, mais qui se lit en filigrane dans son texte, c'est qu' aux trois phases de décatholicisation correspondent trois phases du catholicisme lui-même, trois visages qu'il a fallu adopter, puis abandonner, «trois catholicismes », pourrait-on dire. La « laïcisation » correspond à ce que j'appellerai le catholicisme $d u$ catéchisme, catholicisme de conformité doctrinale, auquel la pratique du catéchisme, sans doute plus encore que la prédication dominicale offrit un formidable réseau de diffusion et qui visa à étendre son littéralisme à tous les niveaux de la pensée religieuse. Ce catéchisme a parfaitement coexisté avec la laïcité tant que son ambition ne dépassait pas celle de fournir un soubassement à la formation morale. La «sécularisation interne » fut à la fois produit et processus d'autodestruction du Catholicisme d'Action Catholique. Celui-ci, issu d'une stratégie du clergé, faisant leur part aux laïcs, a répondu en grande partie aux aspirations de ceux-ci. En acclimatant sans doute un catholicisme plus humain et plus «social», il faisait perdre à celui-ci sa spécificité. De quel catholicisme l' «ultramodernité » est-elle corrélative ? Après une phase politique où les diverses branches de l'Action Catholique avaient pris des structures de parti (fournissant par là à la Résistance une couverture idéale) on recherche un climat plus fraternel, en accord avec l'air du temps. C'est l'âge des communautés. L'archétype des «premiers chrétiens » se marie alors volontiers avec les modèles utopiques de type Buchez ou Barbut. On constitue des communautés à tous les étages, quartiers, paroisses, diocèses (l'Église même ne serait-elle pas une vaste «communauté », une «communion», comme on dit volontiers en contrant l'expression «peuple de Dieu » qui prévalut à Vatican II ?). On rejoint d'une certaine façon un mouvement plus vaste qui fait résulter l'appartenance religieuse d'un jugement de goût, d'un choix entre diverses spiritualités (Berger et Luckman). La question se pose donc maintenant de savoir si le catholicisme de communauté a épuisé ses potentialités, ou s'il est lui-même un avatar de La Religion en miettes (Danièle Hervieu-Léger, Paris, Calmann-Lévy, 2001 [cf. Arch. 118, pp. 29-44]). 


\section{Un tournant anthropologique}

Que nous arrive-t-il depuis trente ans ? C'est au fond la question à laquelle D.H.-L. a voulu répondre dans ce livre, qui traite de la «transformation anthropologique silencieuse » (1) dans laquelle nos sociétés sont entrées au milieu des années 1970, au moment où s'achevaient les Trente Glorieuses. Sans doute y est-il d'abord question du catholicisme, mais l'ouvrage s'inscrit dans une conjoncture intellectuelle plus vaste, où la sociologie des religions ne se sépare pas fondamentalement $\mathrm{du}$ reste des sciences humaines. Cette convergence fait l'objet de la première partie de la note qui suit ; je reviendrai ensuite sur le concept d' « exculturation du catholicisme », qui est au centre de l'ouvrage ; historien moi-même, je terminerai par quelques réflexions sur l'apport de ce livre à une histoire contemporaine du fait religieux en France.

\section{Un nouveau rapport au monde}

Cette transformation de notre rapport au monde tient à trois événements brièvement présentés à la fin du second chapitre. J'en retiendrai deux, le troisième, qui est la mondialisation envisagée comme « la transformation du rapport au temps et à l'espace qui caractérise des sociétés ayant accédé, dans tous les domaines, aux moyens d'une instantanéisation des communications à l'échelle globalisée de la planète » (p. 87), restant davantage en lisière du livre.

D'une part, nous vivons au temps de « l'affirmation de la composante psychologique de la modernité, qui donne à l'individualisme contemporain sa tonalité particulière » (p. 86). Les implications variées de cette entrée dans l'ère de l'individu sont bien connues: que l'on pense, parmi d'autres, aux travaux de Marcel Gauchet sur la sortie de la religion, à ceux d'Ulrich Beck sur la société du risque ou de Daniel Ehrenberg sur la dépression (2). L'un des premiers en France, Henri Mendras, avait retracé dans La Seconde Révolution française les débuts de cette histoire, entre 1965 et 1984 (3). Pour le dire brièvement : l'émergence contemporaine d'un sujet libre au regard des besoins élémentaires, autonome au regard des morales imposées, loin de constituer un confort ou une renonciation au sens, accroît les exigences éthiques qui pèsent sur chacun, tandis qu'elle délégitime les institutions traditionnellement chargées de donner sens à l'aventure commune. Cette déstabilisation des systèmes de valeurs affecte au premier chef l'Église catholique dont elle met en cause le magistère et le système d'emprise. Au quatrième chapitre, D.H.-L. montre par exemple le « désajustement culturel », dans une société d'abondance, d'une pastorale qui faisait fond sur «la peur de manquer » et construisait

(1) J'emprunte l'expression à Paul ZAWADZKI, dans l'ouvrage collectif qu'il a dirigé sous le titre Malaise dans la temporalité, Paris, Publications de la Sorbonne, 2002, p. 11.

(2) Marcel Gauchet, La religion dans la démocratie. Parcours de la laïcité, Paris, Gallimard, 1998 (cf. Arch. 116, pp. 41-52); Ulrich BECK, La société du risque. Sur la voie d'une autre modernité, Paris, Aubier, 2001 ; Daniel Ehrenberg, La fatigue d'être soi. Dépression et société, Paris, Odile Jacob, 1998.

(3) Henri Mendras, La Seconde Révolution française 1965-1984, Paris, Gallimard, 1988. 
l'expérience religieuse autour des notions autrefois communes de faim et de soif. On pourrait lui objecter que les mêmes notions peuvent être réinvesties d'une signification nouvelle, ordonnées au dépassement de soi dans un contexte communautaire, comme en témoigne le succès du jeûne du Ramadan parmi les musulmans français ; et que la conception de la foi comme expérience de guérison, centrale chez les charismatiques, reprend à son compte l'exigence de santé parfaite qui se profile comme la nouvelle utopie de nos sociétés riches (4). Mais on la suit très volontiers lorsqu'elle montre combien le « repli de l'idéal d'accomplissement sur une conception purement individualiste de l'autoréalisation » (p. 165), qui caractérise nos sociétés hypermodernes, met à mal la prédication chrétienne du bien commun. Après tout, ne tient-on pas là une des contradictions auxquelles s'est heurté l'aggiornamento conciliaire, après 1965, pour s'être rallié à la modernité sur la base d'un humanisme communautaire et militant au moment même où nos sociétés basculaient du côté de l'individu et du désir d'épanouissement de soi (5) ?

Le tournant anthropologique dont il est ici question tient aussi à « la transformation qualitative du rapport à la nature qu'induisent les avancées fulgurantes de la science et des technologies, en particulier dans les domaines des sciences du vivant et des neurosciences » (p. 87). Á l'inverse de la mondialisation, ce dernier point me paraît essentiel pour comprendre le livre. On peut renvoyer le lecteur aux dernières pages du troisième chapitre, lorsque l'auteure s'interroge sur les effets culturels de la rupture du lien entre nature et agriculture qu'entraînent les techniques nouvelles de la biogénétique ; et plus encore au chapitre VI, intitulé «Quand la nature n'est plus un ordre », où elle analyse les enjeux sociaux des nouvelles techniques de manipulation du vivant. Quelles sont les conséquences de notre capacité technique à maîtriser les processus liés à la vie (procréation assistée, thérapie génique) et à la mort (soins palliatifs, problèmes de définition de la mort, voire de son administration à travers la question de l'euthanasie) (6) ? Comment penser le fait que l'application à l'homme de la génomique est susceptible de se traduire, selon le mot de Jürgen Habermas dans sa polémique avec Peter Sloterdijk, par « la disparition de la frontière entre la nature, que nous sommes, et l'appareil organique, que nous nous donnons » (7) ? Or cette frontière, à la fois juridique et philosophique, mais aussi étroitement liée à l'évolution de la société technique, a longtemps été un fondement essentiel du partage entre sacré et profane. Et c'était bien à l'héritage chrétien que nous demandions d'en rendre raison.

D.H.-L. s'est attachée ainsi à la diagonale qui conduit l'Église catholique de la condamnation de la contraception et de l'avortement au refus de la procréation médicale assistée. Elle montre que ce qui est en jeu, au fond, c'est le déphasage entre notre expérience technique du monde et un ordre biologique de la nature dont l'Église s'est voulue -et se veut toujours- solidaire. Elle montre aussi combien les débats récents sur le PACS et l'homoparentalité constituent un enjeu essentiel de la sortie de la famille hors de l'ordre naturel sacralisé par l'Église, comment ils font le

(4) Voir en particulier Lucien SFEZ, dir., L'utopie de la santé parfaite, Paris, PUF, 2001.

(5) Je me permets de renvoyer sur ce point au premier chapitre de mon livre, La crise catholique. Religion, société, politique en France (1965-1978), Paris, Payot, 2002 (cf. Arch. 120.30).

(6) Voir Dominique Memmi, Faire vivre et laisser mourir. Le gouvernement contemporain de la naissance et de la mort, Paris, La Découverte, 2003.

(7) Jürgen Habermas, L'avenir de la nature humaine. Vers un eugénisme libéral ?, Paris, Gallimard, 2002, p. 39. 
lien, de ce point de vue, entre «l'avènement moderne du sujet autonome, législateur de lui-même» et «la révolution contemporaine du rapport à la nature » (pp. 248-249). Elle rejoint alors, me semble-t-il, les réflexions inspirées à la juriste Marcela Iacub par l'arrêt Perruche, les polémiques qu'il a suscitées et ce qu'elles disent de la transformation récente par le droit de la notion, longtemps pensée sur un mode humaniste et chrétien, de personne humaine (8). En se confrontant à la polémique sociale, la sociologie devient une pratique de l'engagement critique.

\section{L'exculturation du catholicisme}

Le lecteur l'aura compris : un des intérêts principaux de ce livre réside dans la manière dont le fait religieux sert de porte d'entrée à une analyse plus ample des mutations du lien social et du rapport au monde. Le destin du catholicisme en France y prend place à travers le concept d' «exculturation». De quoi s'agit-il au juste, au-delà de l'inversion d'une notion qui fit les beaux jours de la pensée missionnaire ? D.H.L. part du constat que si la crise est depuis au moins deux siècles la forme normale du rapport du catholicisme à la modernité, l'originalité de celle qu'il traverse depuis une trentaine d'années loge quelque part entre l'atonie du débat interne (c'est la «paix des cimetières ») et le sentiment d'une « ringardise catholique », les catholiques se plaignant moins des attaques qu'ils subissent que $\mathrm{du}$ « mépris - plus souvent apitoyé qu'agressif, dont ils ont le sentiment d'être environnés du seul fait de leur appartenance affichée à une institution dont on moque la disqualification culturelle plus encore qu'on ne dénonce (...) la prétention à dire le vrai et le bien pour la société entière » (p. 11).

L'exculturation du catholicisme, c'est donc « la possible déliaison de l'affinité élective que l'histoire a établie en profondeur entre les représentations partagées des Français (la «culture» qui leur est commune) et la culture catholique » (p. 97) ; c'est aussi le fait que « la mutation culturelle que nous sommes en train de vivre et qui bouleverse de fond en comble l'armature symbolique de notre société est susceptible de produire un évidement peut-être ultime du catholicisme en son sein » (p. 20) ; ce qu'elle met en jeu, c'est encore «la capacité du catholicisme à nourrir et à alimenter -au-delà des constructions individuelles du sens qui continuent de se référer au corps de croyance et aux valeurs que l'Église affiche et promeut- des significations collectivement partagées, dont la source religieuse puisse demeurer identifiable dans la société française d'aujourd'hui » (p. 131). Autrement dit, le catholicisme n'est plus le moteur de la crise qu'il traverse, comme s'il subissait ainsi les ultimes conséquences d'un processus de marginalisation puis d'éviction hors du champ des évidences collectives.

Déliaison, épuisement, évidement... Le vocabulaire le montre : ce livre est une réflexion sur la disparition et la perte, qui ne va pas sans quelque effet de nostalgie ou de tragique, même si l'auteure s'en défend (« la fin d'un monde n'est pas la fin du monde », conclut-elle sans doute pour consoler ses lecteurs chagrins...). De deux manières au moins, ce constat me paraît nourrir une réflexion ou un retour sur le

(8) Marcela Iacub, Penser les droits de la naissance, Paris, PUF, 2002. 
travail de la sociologie des religions. Sociologie en creux, d'une part, sur les implications historiques sur laquelle je reviendrai plus loin: en se retirant, le catholicisme dit quelque chose des lieux qu'il abandonne à leur autonomie, la ruralité ou la famille, la technique ou le vivant. En retour, ce livre parle moins du catholicisme en tant que tel que de ce que nous en apprennent ces mêmes lieux au moment où il les déserte. Il y a là comme la poursuite, au-delà de l'exculturation, au prix d'une sorte de «passage par le négatif », de la réflexion durkheimienne sur la fonction sociale du religieux.

Sociologie du manque, également, qui me paraît faire de ce livre l'aboutissement raisonné d'un itinéraire intellectuel commencé avec l'analyse des mouvements communautaires post-68 (9). On sait ce que l'histoire du catholicisme, et en particulier l'idée de réforme qui en est un moteur, doit à l'utopie communautaire, depuis les ordres mendiants du Moyen-Âge jusqu'aux communautés de base où s'inventait le «christianisme critique » des années 1970. On sait aussi ce que la sociologie française des religions doit au fait communautaire, ne serait-ce qu'à travers l'expérience qu'en firent ses fondateurs et comment ils la réinvestirent dans leur travail de chercheur -je pense ici, en particulier, à l'héritage d'Henri Desroche (10). Mais la communauté est aussi une institution fragile, qui n'existe que dans sa propre tension à disparaître, dans l'épreuve du manque qui la taraude, parce qu'elle est à la fois une utopie et une forme de sociabilité où s'éprouve l'échec de cette utopie (11). Bataille, Blanchot l'avaient bien compris, au temps d'Acéphale et du Collège de sociologie; Jean-Luc Nancy en a repris naguère l'analyse : l'expérience de la communauté, c'est l'expérience qu'il n'y a pas de communauté, qu'elle s'est déjà dissoute dans le moment où l'on croit la saisir pour en parler (12). Est-ce beaucoup solliciter ce livre que d'y voir la pointe ultime d'une réflexion marquée par l'expérience initiale de la perte, une expérience que D.H.-L. n'a cessé depuis lors d'interroger comme le fait majeur du catholicisme contemporain, depuis le paradigme de la sécularisation jusqu'à celui de l'exculturation, en passant par le déracinement des pratiques et la question d'une mémoire religieuse dont la transmission serait devenue problématique (13) ?

Enfin, l'étude de ce processus d'exculturation met en relief ce que D.H.-L. appelle le «travail civilisationnel » du catholicisme, dans des chapitres très éclairants pour une histoire politique du catholicisme français contemporain. Il importe en effet de rappeler que ce livre se limite au cas français, ce qui renvoie à l'originalité de notre laïcité républicaine. Si la politique est l'instance de fabrication et de

(9) Danièle Hervieu-LÉGer, De la mission à la protestation. L'évolution des étudiants chrétiens en France (1965-1970), Paris, Cerf, 1973 ; Danièle Hervieu-Léger, Bertrand Hervieu, Des communautés pour les temps difficiles. Néo-ruraux ou nouveaux moines, Paris, Centurion, 1983.

(10) Voir le chapitre que lui consacre Danièle Hervieu-LÉGER dans Sociologies et religion. Approches classiques (en collaboration avec Jean-Paul Willaime), Paris, PUF, 2001, pp. 263-289 (cf. Arch. $122.25)$.

(11) Denis Pelletier, «Utopie communautaire et sociabilités d'intellectuels en milieu catholique dans les années quarante », Les Cahiers de l'IHTP, no 20, mars 1992, pp. 172-187.

(12) Maurice Blanchot, La communauté inavouable, Paris, Éditions de Minuit, 1983 ; Jean-Luc NANCY, La communauté désœuvrée, Paris, Christian Bourgois, 1986.

(13) Danièle HERVIEU-LÉGer, Françoise CHAMPION, Vers un nouveau christianisme ? Introduction à la sociologie du christianisme occidental, Paris, Cerf, 1986 ; Danièle HERVIEU-LÉGER, La religion pour mémoire, Paris, Cerf, 1993 (cf. Arch. 84.47) ; Le pèlerin et le converti. La religion en mouvement, Paris, Flammarion, 1999 (cf. Arch. 108.50). 
validation des normes et des valeurs communes, alors l'hypothèse d'une exculturation récente du catholicisme suggère a contrario d'approfondir ce qu'il en fut avant, c'est-à-dire de quelle nature fut le rapport entre la République et le catholicisme au temps où ce dernier était en situation de produire du sens pour tous. Historiens du religieux, nous avons trop facilement tendance à penser ce rapport sur le mode de l'exclusion réciproque. C'est oublier que, sur des terrains aussi essentiels que ceux de la ruralité ou de la famille, qui font chacun l'objet d'un chapitre du livre, le catholicisme a tenu une place non négligeable dans l'élaboration d'une identité collective qui fut à la fois républicaine et française, ordonnée à l'universel et à l'enracinement, entre patrie des droits de l'homme et juxtaposition des petites patries, entre l'esprit de Valmy et l'exaltation du soldat-paysan, entre les droits de l'homme et l'agrarisme à courte échelle.

Disons-le autrement : en soulignant, par exemple, « la convergence paradoxale (et jusqu'à un certain point contre nature) qui s'est établie entre la vision conservatrice d'une "spécificité paysanne », noyau dur de l'ordre social et religieux à préserver, et l'idéologie paysanniste de la République, pourtant enracinée dans la vision promue par la Révolution du «paysan-citoyen»»(p. 102), D.H.-L. nous rappelle qu'il y a un contenu anthropologique de la République, que le catholicisme a largement contribué à façonner par son travail civilisationnel, en amont de l'affrontement qui opposa France cléricale et France laïque. Une telle analyse rejoint celles qu'a menées l'historien américain Herman Lebovics sur la «Vraie France », en exhumant la figure de Louis Marin (1871-1960), ce grand parlementaire de droite, catholique et libéral, qui fut un ministre républicain mais aussi le président de plusieurs sociétés savantes où s'élabora le programme de l'ethnologie française (14). En suggérant que les catholiques ont ainsi contribué à la construction culturelle du modèle républicain, en dépit de leur mise à l'écart des lieux de décision, ce livre propose quelques outils dont les historiens soucieux d'ouvrir l'histoire de la France contemporaine à une anthropologie du politique pourraient faire leur miel.

\author{
Denis PELLETIER \\ Laboratoire de Recherches Historiques en Rhône-Alpes - CNRS \\ Université Lumière Lyon 2
}

(14) Herman Lebovics, La «Vraie France ». Les enjeux de l'identité culturelle, 1900-1945, Paris, Belin, 1995. 


\section{Catholicisme la fin d'un monde : a British perspective}

I read this book during a recent visit to Paris - each experience enhanced the other as I understood more of the changes taking place both in France and in the French Catholic Church. Catholicisme, la fin d'un monde is best described as an extended (and provocative) essay on the state of Catholicism in France at the turn of the millennium. For a British reader a series of questions immediately present themselves. To what extent are the same things happening to the mainstream churches in British society? Are they happening for the same reasons? And will the consequences be similar or different? Or to put the same point in a different way: to what extent do the facts and argument of this book endorse the specificity of the French case or to what extent can they be extrapolated to other European/modern societies?

Before engaging these questions, it is important to place this book in the trajectory of Hervieu-Léger's own work. The book is the last of a trilogy, which in itself forms part of a distinguished publishing career. The earlier volumes in the trilogy are Le pèlerin et le converti. La religion en mouvement (Paris, Flammarion, 1999) and La religion en miettes ou la question des sectes (Paris, Calmann-Lévy 2001) The first considers the new forms of religious activity that are emerging in modern France, as indeed elsewhere, as the older patterns erode: modern individuals seek and search (they are pilgrims); and increasingly they make definitive decisions (they convert). Gone, or going, are the stable forms of religious life in which individuals can be safely classed as pratiquants or not, with the expectation that they will remain in the categories in which they started out. The discussion of sects - and here the context is specifically French - focuses on the incapacity of existing structures (both religious and secular) to deal with the new forms of belief that are emerging in France.

Catholicisme, la fin d'un monde completes the picture in that it describes in detail the collapse not only of the structures of the French Catholic Church but of the culture that underpinned these. The process, in fact, is one of a progressive disembedding of the Catholic Church from both French society and French culture. This process is described over time (Chapter 2), with respect to space (Chapter 3) and in terms of a range of cultural themes (Chapters 4, 5 and 6).

Each of these ideas requires expansion. Chapter 2, for example, offers three ways into the somewhat stormy relationship between the Catholic Church and modern French society. The first describes the clash between the vision of the world represented by the Church on the one hand and by the Revolution on the other. No country in Europe experienced this clash in quite the same way, a fact that inevitably coloured everything that followed: i.e. the implications of both modernity (the second approach) and ultra-modernity (the third approach) for the life of the French Church, both internally and in its relationship with the wider society.

The following chapter draws from Hervieu-Léger's extensive knowledge of rural France. It evokes both the natural affinity between traditional patterns of rural life and the Catholic Church in its historic forms, and the acute difficulties of the latter when the twin processes of industrialisation and urbanisation hit France not only relatively late, but correspondingly fast. The embeddedness, both structural and cultural, of the historic churches of Europe in a pre-industrial and predominately rural way of life is the crucial point. With this in mind, secularisation theorists are entirely correct to concentrate their analyses on the collapse of this pre-industrial world: it was a shock from which the historic churches of Europe never fully recovered. 
The three chapters that come next embark on innovative themes : they are concerned with more recent and primarily cultural challenges to Catholicism in France. A late modern emphasis on personal fulfilment sits uneasily alongside traditional Catholic teaching. Family life, moreover, has evolved in ways that bewilder the Church and challenge both its dogma and its policies; so too the profound shifts in the understanding of life and death as advanced medical techniques push the boundaries ever further. The parameters of life itself (birth and death), in which the churches have played such a major part, are themselves subject to change.

What then emerges? New forms of religious life can be found (the pilgrim and the convert for example). It is important to remember, moreover, that the Catholic Church is not the only institution in trouble as the pressures of modernity exert their force: secular institutions and secular authority are equally at risk (pp. 271275). Traditional formulae are eroded in both spheres as patterns of obligation give way to patterns of consumption. Modern individuals find their own ways forward, motivated more by the choices available than by a set of obligations or requirements. It follows that those institutions most closely associated with obligation (not least the churches) feel the pinch most sharply.

That is equally so in other modern societies. In what ways then is the French case indicative of broader changes and in what ways is it exceptional? The question can be asked from two rather different vantage points - within Europe and without. From Britain, for example, the picture or the process described by D.H.-L. is entirely recognisable: the Church of England and the Church of Scotland were deeply embedded in pre-industrial, rural society and felt the onset of industrialisation keenly. The process, however, started earlier and took much longer leading to a more gradual shift (or, if you like, a lower level of pain, but experienced for longer). A second point is rather different: the Protestant churches of northern Europe had already undergone change and from the inside - driven this time by different theological understandings. The institutional collapse was, therefore, of a different order. In the French case, everything happens at once and in a particularly dramatic way: the aftershock is correspondingly severe.

A further contrast comes from the United States. Here neither Catholic nor Protestant churches had time to embed themselves into the pre-industrial order before the onset of industrialism. Religious congregations of all kinds moved easily into the growing cities of industrial America. The cultural tensions were also very different as the implications of the enlightenment worked themselves out in positive rather than negative ways in their applications to religion. Hence an entirely different scenario in a society where modernity and religion become mutually reinforcing categories rather than necessarily opposed. In this respect, Hervieu-Léger's analysis, rather like French wine, does not travel well.

It deserves, however, to be widely read and not only by French Catholics and French scholars of religion. And with this in mind, I - as an English reader - long for a bibliography and an index. I appreciate that this is a provocative essay rather than a work of reference. Its essence derives however from wide reading and draws from a rich literature. It would be good if the latter were more directly accessible to the fortunate readers of this book, whoever and wherever they may be. 